\title{
Phyto- und Mykotoxine (2)
}

\author{
Amygdalin und andere cyanogene Glycoside \\ Jean-Michel Jeannin
}

\section{Chemie und Biochemie}

Cyanogene Glycoside sind weitverbreitete Verteidigungssubstanzen von Pflanzen. Es handelt sich dabei um Glycoside, die eine Nitrilgruppe tragen. Durch enzymatischen Abbau wird das Molekül in einen Zucker, in ein Aldehyd und in Blausäure aufgespalten. Die Abspaltung des Zuckers wird von der $\beta$-Glycosidase, die Abspaltung des Cyanids vom Aldehyd durch das Enzym Nitrilase vermittelt. In der intakten Pflanze liegen die cyanogenen Glycoside und die Glycosidase in getrennten Kompartimenten vor. Typische, häufig vorkommende cyanogene Glycoside sind das Amygdalin (Abb. 1), Prunasin (D-Mandelonitril) (Abb. 2) und Sambunigrin (L-Mandelonitril) (Abb. 3).

\section{Toxikologie}

Die orale mittlere letale Dosis $\left(\mathrm{LD}_{50}\right)$ der Blausäure beträgt beim Kaninchen $2,7 \mathrm{mg} / \mathrm{kg}$, beim Meerschweinchen $3 \mathrm{mg} / \mathrm{kg}$, bei der Maus $4,2 \mathrm{mg} / \mathrm{kg}$ und bei der Ratte $10-15 \mathrm{mg} / \mathrm{kg}$. Für den Menschen sind $1 \mathrm{mg} / \mathrm{kg}$ Blausäure bzw. 2 mg Kalium- oder Natriumcyanid tödlich (Tab. 1). Pflanzenteile, die $200 \mathrm{mg}$ Blausäure pro kg Feuchtgewicht enthalten, gelten als potenziell toxisch. Die in den Blättern der Pflanzen enthaltenen cyanogenen Glycoside sind wegen ihrer geringen Menge toxikologisch unbedenklich, nicht aber die in den Samen enthaltenen Mengen: 1 Bittermandelsamen pro kg Körpergewicht (KG) ist für den Erwachsenen tödlich, ebenso $1 \mathrm{~g}$ zerquetschte Apfeloder Birnenkerne pro kg KG. Bei falscher Zubereitung kommen auch Vergiftungen durch die Süssmandel (Prunus dulcis) vor, die einen gewissen Anteil bitterer Kerne enthält.

Blausäure wird mittels freier Diffusion über den Magen-Darm-Trakt oder über die Haut rasch aufgenommen und verteilt. In der Zelle bildet die Blausäure mit dem dreiwertigen Eisen $\mathrm{Fe}^{3+}$ bestimmter Enzyme, unter an-

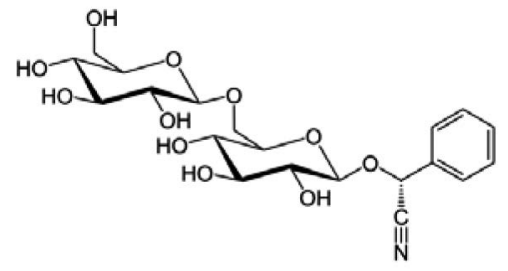

Abb. 1. Amygdalin.
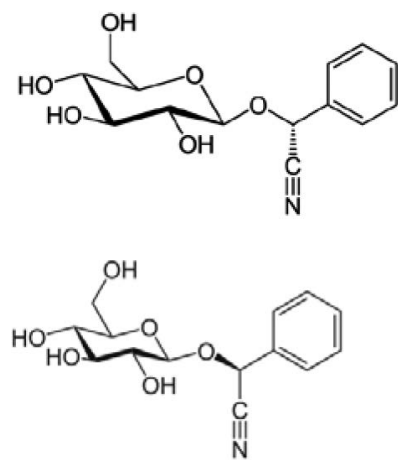

Abb. 2. Prunasin

Tab. 1. Giftklassen der WHO (nach [1])

\begin{tabular}{lll}
\hline Klasse & Bezeichnung & LD $_{50}$ Ratte, $\mathrm{mg} / \mathrm{kg} \mathrm{KG}$ \\
\hline Ia & äusserst giftig & $\leq 5$ \\
Ib & sehr giftig & $5-50$ \\
II & giftig & $50-500$ \\
III & schwach giftig & $>500$ \\
\hline
\end{tabular}

derem mit der Cytochromoxidase, einen Komplex. Die Hemmung der Cytochromoxidase hat eine unmittelbare Beendigung der Zellatmung zur Folge. Die aus der Atmung gewonnene Energie kann nicht mehr gespeichert und für die Stoffwechselprozesse der Zelle zur Verfügung gestellt werden. Es kommt zu einer Akkumulation von Lactat, was zu einer Lactatazidose führt. Besonders empfindlich sind die energieintensiven Prozesse des Gehirns betroffen; der Tod tritt ohne Behandlung infolge einer Atemlähmung ca. 30 min nach Aufnahme des Gifts ein. Das Enzym Rhodanase wandelt die Blausäure in das ungiftige Rhodanid um. Der Prozess kann durch die Verabreichung von Thiosulfat beschleunigt werden.

\section{KARGER}

Fax +497614520714 Information@Karger.com www.karger.com 
Durch eine intravenöse Verabreichung von $\mathrm{Fe}^{3+}$ kann eine Blausäurevergiftung schneller überwunden werden, da die Bildung des Blausäure-Eisen-Komplexes reversibel ist [1].

\section{Vergiftung und Notfallmassnahmen}

Die Symptome einer Vergiftung äussern sich in Form von Schleimhautreizung, Gesichtsrötung, Atembeschwerden, Kratzen im Rachen und Atemnot. In schweren Fällen führt eine Vergiftung zum Tod. Die Notfallbehandlung besteht aus dem Auslösen von Erbrechen und Magenspülung bzw. alternativ der intravenösen

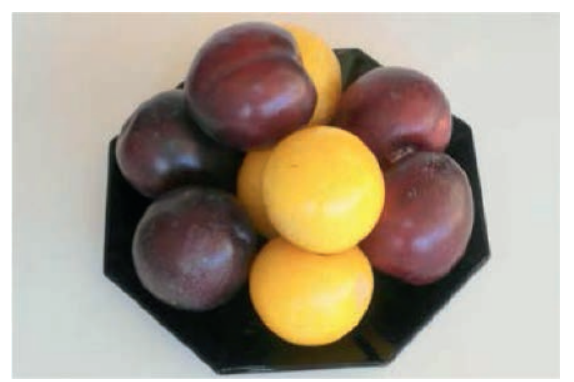

Abb. 4. Gelbe und rote Pflaumen.

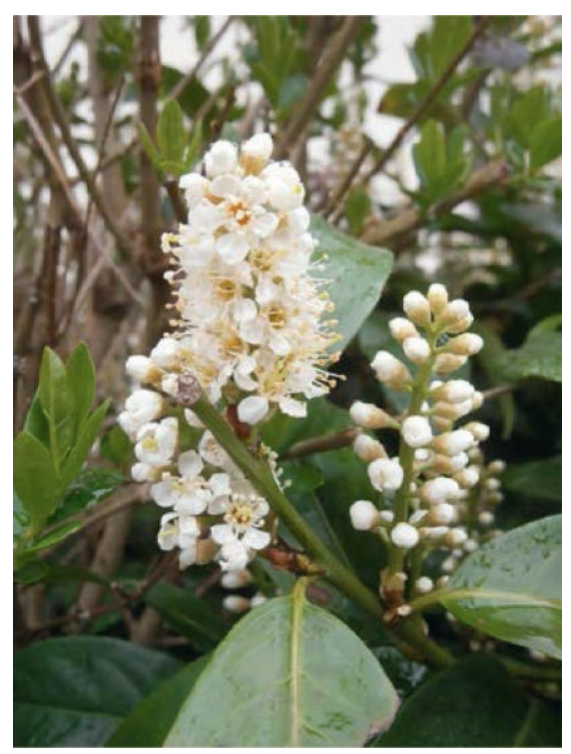

Abb. 5.

Lorbeerkirsche. Basel, 2013.

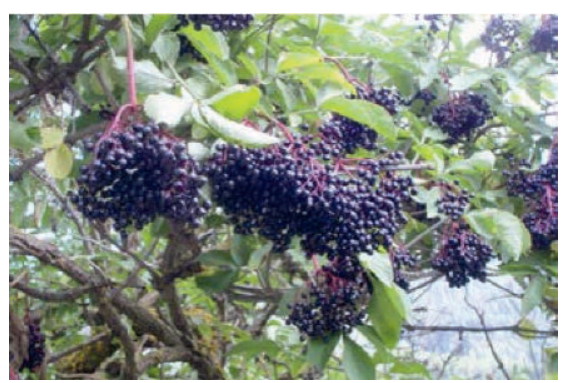

Abb. 6.

Holunderbeeren. Schuls, 2007.
Gabe von Natriumthiosulfat. In schweren Fällen ist eine Intubation zur Sauerstoffbehandlung angezeigt; eine allfällige Lactatazidose wird mit Natriumbicarbonat oder Tris-Puffer (Tris(hydroxymethyl)-aminomethan) behandelt. Auf die Entstehung eines Lungenödems muss geachtet werden [1].

\section{Vorkommen}

Cyanogene Glycoside kommen besonders häufig bei Rosaceen vor, worunter die Steinfrüchte Aprikose (Prunus armeniaca), Bittermandel (P. amygdalis amara), Pflaume (P. domestica) (Abb. 4), Lorbeerkirsche (P. laurocerasus) (Abb. 5), Sauerkirsche (P. cerasus), Süsskirsche (P. avium), Süssmandel (P. dulcis), Zwetschge (Prunus domestica subspec. domestica), aber auch die Kernfrüchte Apfel (Malus domestica), Birne (Pyrus communis) und Quitte (Cydonia oblonga) gehören. Cyanogene Glycoside kommen auch im Holunder (Sambucus nigra) (Abb. 6) oder in der Hirse vor [1]. Nachfolgend werden zwei der Pflanzenarten, die cyanogene Glycoside enthalten, vorgestellt.

\section{Prunus laurocerasus}

Die Lorbeerkirsche (syn. Kirschlorbeer) ist ein immergrüner, bis $4 \mathrm{~m}$ hoher Strauch aus der Familie der Rosengewächse (Rosaceae). Die Blätter sind länglich, ledrig und glänzend. Die weissen Blüten stehen in aufrechten Trauben; die Früchte sind kleine, schwarzviolette, kirschenartige Steinfrüchte. P. laurocerasus ist in Südosteuropa und in Kleinasien heimisch. Der Kirschlorbeer gehört zur Giftklasse II [1].

Inhaltsstoffe: Die Blätter enthalten 1-1,5\% Prunasin, die Samen 0,16-0,21\% Amygdalin, das Fruchtfleisch 100-200 ppm Blausäure [1]. Wird das Prunasin isoliert, wird ein Teil in das Isomer Sambunigrin umgewandelt. Das so erhaltene Racemat wird als DL-Mandelonitrilglycosid oder Prulaurasin bezeichnet [2]. Weitere Bestandteile sind Ursolsäure (1\%), Wachse, Tannine und Emulsine [1].

Verwendung: Kirschlorbeerwasser (Aqua laurocerasi) wurde zum Aromatisieren von Heilmitteln, z.B. Hustensirup, und von Lebensmitteln, z.B. Bonbons [2], sowie als Atmungsstimulans verwendet [1], ferner (aus der Sicht der amerikanischen Autoren) als Sedativum, Antineuralgikum und Spasmolytikum [2]. Volksheilkundlich werden die Blätter als Antitussivum, Schlafmittel, Mittel gegen Magen- und Darmkrämpfe und als Antiemetikum eingesetzt [2]. Kirschlorbeerwasser enthält 0,1\% Blausäure und wurde (wird?) für Mord und Selbstmord verwendet [1]. 


\section{Sambucus nigra $\mathrm{L}$.}

Der Schwarze Holunder ist ein 3-7 m hoher Strauch aus der Familie der Geissblattgewächse (Caprifoliaceae). Die Rinde ist warzig und riecht unangenehm. Die Blätter sind unpaarig gefiedert und stehen gegenständig. Die weissen Blüten stehen in flachen Trugdolden. Blütezeit ist von Mai bis Juli. Die Früchte sind schwarze bis schwarzviolette Beeren, die der Strauch im Herbst trägt [3].

Inhaltsstoffe: Die Blüten enthalten ca. 0,3\% ätherische Öle, hierunter Alkane, Triterpene, Ursolsäure, 30 $\beta$ Hydroxyursolsäure, Oleanolsäure, Sterole, Quercetin, Kaempferol, Isoquercitrin, und Rutin (bis zu 1,9\%). Die Blätter enthalten Sambunigrin, Cholin, Rutin, Quercetin, Sterole, Triterpene, Alkane, Fettsäuren, Tannine, Harze, Fette, Zuckerarten und Ascorbinsäure [2].

Verwendung: Arzneilich werden die Blüten, die Blätter, die Rinde und die Früchte verwendet. Die wichtigste
Anwendung erfolgt in Form eines Tees aus den Blüten als schweisstreibendes Mittel bei Erkältungskrankheiten. In der Homöopathie wird S. nigra aus frischen Blüten und Blättern zubereitet. Das Mittel wird bei Schnupfen und zur Aktivierung der körpereigenen Abwehr sowie auch gegen Asthma bei Kindern verabreicht. In der Volksmedizin werden Zubereitungen aus den Beeren, den Blüten und auch der Rinde für verschiedenste Indikationen breit angewendet: Infektionskrankheiten (Tee aus den Blüten), Rheuma (Blüten, Blätter, Rinde), Erkältungskrankheiten/zur Abführung (Saft der Beeren, Rinde). Wird die Rinde von oben nach unten abgeschabt, ist sie ein Abführmittel; wird sie von unten nach oben abgeschabt, gilt sie als Brechmittel [3].

Unerwünschte Wirkungen: Unreife Beeren sind schwach giftig. Der Konsum des rohen Safts der Beeren kann zu Übelkeit, Erbrechen und Durchfall führen [3].

\section{Literatur}

1 Wink $M$, et al: Handbuch der giftigen und psychoaktiven Pflanzen. Stuttgart, WVG, 2008.
2 Leung AY, Forster S: Encyclopedia of Common Natural Ingredients, ed 2. New York, Wiley, 1996.
3 Holunder; in Pahlow M: Das grosse Buch der Heilpflanzen. Augsburg, Bechtermünz, 2000. 